\title{
How Can Studies of Animals Help to Uncover the Roles of Genes Implicated in Human Speech and Language Disorders?
}

\author{
Simon E. Fisher
}

\section{Summary}

The mysterious human propensity for acquiring speech and language has fascinated scientists for decades. A substantial body of evidence suggests that this capacity is rooted in aspects of neurodevelopment that are specified at the genomic level. Researchers have begun to identify genetic factors that increase susceptibility to developmental disorders of speech and language, thereby offering the first molecular entry points into neuronal mechanisms underlying human vocal communication. The identification of genetic variants influencing language acquisition facilitates the analysis of animal models in which the corresponding orthologs are disrupted. At face value, the situation raises a perplexing question: if speech and language are uniquely human, can any relevant insights be gained from investigations of gene function in other species? This chapter addresses the question using the example of FOXP2, a gene implicated in a severe monogenic speech and language disorder. FOXP2 encodes a transcription factor that is highly conserved in vertebrate species, both in terms of protein sequence and expression patterns. Current data suggest that an earlier version of this gene, present in the common ancestor of humans, rodents, and birds, was already involved in establishing neuronal circuits underlying sensory-motor integration and learning of complex motor sequences. This may have represented one of the factors providing a permissive neural environment for subsequent evolution of vocal learning. Thus, dissection of neuromolecular pathways regulated by Foxp 2 in nonlinguistic species is a necessary prerequisite for understanding the role of the human version of the gene in speech and language.

Key Words: Speech; language; FOXP2; evolution; vocal learning; verbal dyspraxia; Broca's area; caudate nucleus; cerebellum; song system.

\section{INTRODUCTION}

For many years, scientists have been intrigued by the aptitude of human children for acquiring highly intricate communication skills with little effort and without any need for formal instruction. A prevailing view is that this extraordinary capacity for imbibing language depends on particular features of brain structure and/or

From: Contemporary Clinical Neuroscience: Transgenic and Knockout Models of Neuropsychiatric Disorders Edited by: G. S. Fisch and J. Flint @ Humana Press Inc., Totowa, NJ 
neurological processing that are innately encoded (1). This position is supported by a wealth of indirect evidence from a diverse range of sources, including identification of so-called universal aspects of linguistic structure $(2,3)$, data from neuropsychological studies of language acquisition (4-6), adult cases of disrupted speech and/or language caused by brain lesions in particular regions (7-9), mapping of functionally active neural sites during language-related tasks (10-12), and comparisons between our species and other primates, who, despite being closely related, do not share the same capacities for vocal learning (13-15). In the search for more direct evidence that might support a role for genetic factors in this most elusive of human skills, some researchers have recently begun to focus on studies of the small (but significant) proportion of children who are unable to acquire normal speech and language abilities, although they possess adequate intelligence and are exposed to the usual level of linguistic input in their environment (16). These cases of developmental speech and language disorder may potentially have a variety of causes, but it has clearly been established that a large part of susceptibility is accounted for by genetic influences (17). There is considerable effort being invested worldwide with the intention of precisely identifying allelic variants that predispose to speech and language impairment (17), and other language-related disorders, such as developmental dyslexia (18) and autism (19). Progress has been impeded by complexity at phenotypic and genotypic levels (17-19). As such, despite considerable success in localizing genetic effects to roughly defined chromosomal intervals, it has proved difficult to pinpoint specific allelic variants that are unambiguously involved in disordered language development (17). However, the pace of research is accelerating and it is likely that this situation will be remedied in the course of the next decade.

At present, we know of just one gene whose mutation or disruption is clearly implicated in impaired development of speech and language, while leaving other faculties relatively spared (20). The discovery of this gene, known as FOXP2, has opened up a number of exciting new avenues for exploration of neurogenetic influences on vocal communication (21). One of the most powerful and flexible systems for studying the involvement of a gene in the development and function of the brain is to exploit animal models in which the gene of interest is disrupted (22). However, this leads us to something of a paradox. Speech and language are widely considered to be unique to our species (which is one of the reasons why humans find this research topic so intriguing) (1). How can one effectively study the potential role of a gene in speech and language by investigating its function in a nonlinguistic species? Mice, currently the geneticist's mammal of choice for gene knockouts, are notoriously poor at making conversation, and even worse at conjugating verbs. The problem seems to be pervasive, because we cannot even solve it by moving to our closest evolutionary relatives, other primates (an effective recourse when studying neurodegenerative disease, for example; see ref. 23) and the prospects for investigating gene function during human brain development are severely limited at present, for a host of ethical and technical reasons. 
The aim of this chapter is to persuade the reader that, however counterintuitive this may seem at the outset, studies of gene function in other animals (and even birds) may be critical for yielding insights into innate aspects of vocal communication in humans. The evidence to support this will, by necessity, come predominantly from studies of $F O X P 2$, because this is currently the only known molecular inroad into the relevant pathways. Nevertheless, the FOXP2 findings serve to illustrate some general points that are likely to apply more widely in studies of the genetic bases of human neurodevelopment. Crucially, any informed discussion regarding the potential role of genetic factors in human speech and language depends on a clear definition of what is meant by this term, accompanied by insight into exactly how our communication skills do differ from those of other species.

\section{DISSECTING SPEECH AND LANGUAGE: SHARED VS UNIQUE CHARACTERISTICS}

In the broad sense, the human capacity for speech and language is best viewed as a multicomponential system enabling rapid verbal communication of an infinite array of complex meaningful ideas from one human being to another (24). I am concerned here primarily with vocal communication; considerations of features of written language that developed in the past $2000 \mathrm{yr}$ or aspects of modern forms of sign language are not directly relevant to the present discussion. The average human is able to make very rapid and precisely synchronized movements of articulators (tongue, lips, jaws, and so on), coordinated with time of onset/offset for vibrations of the larynx, to produce the particular sound sequences that are peculiar to speech (25). The combinatorial nature of speech-a finite number of subunits can be assembled to form an unlimited range of unique utterances-is a crucial feature of human vocal communication (1). This is constrained by rules of language (syntax) that govern the ways in which units can be combined, at word, phrase, and sentence levels, to encode the intended meaning (semantics) $(2,3)$. The stream of speech sounds that are produced by the speaker has to be perceived by the auditory system of the listener, and somehow decoded (in a way that is independent of irrelevant factors such as variation in vocal tract anatomy from one speaker to the next), thus, yielding the particular sequence of phonetic units that were originally intended by the speaker (26). The phonetic sequence must then be parsed by the listener, using the same multilevel syntactic rules that would have been adopted by the speaker, and assuming an equivalent vocabulary of lexical items.

It is obvious from this already grossly oversimplified view of the workings of speech and language, that human vocal communication depends on multiple processes and involves a variety of cognitive, motor, and sensory substrates. It should also be clear that, if the system is to be effective in correctly conveying meaning from speaker to listener, each of these processes must be placing substantial constraints on the workings of the others. In other words, the relationships between different elements of the speech and language system must be finely tuned to provide parity, and it is difficult to explain the evolution of human vocal communica- 
tion without assuming a considerable degree of bootstrapping (27). However, there are conflicting opinions regarding the specificity of each of the sensory, motor, and cognitive substrates for the capacity for language (e.g., refs. 24,27). At the heart of this debate, lies the following question: are there aspects of the human vocal communication system that are unique to our species (i.e., one or more qualitatively distinct mechanisms) or do the capabilities of our species just reflect honing of features that are also present in nonspeaking animals (i.e., quantitative variation of pre-existing mechanisms)? To answer this question, scientists have tried to assess whether particular sensory, motor, or cognitive features of the human vocal communication system are present in a comparable form in other animals and/or birds. Comparative studies represent a burgeoning field, therefore, I will confine my discussion to three of the more prominent areas of research: speech production (vocal tract anatomy/motor control), speech perception, and syntactical processing. The emerging findings illustrate that animals and birds, despite their lack of speech and language, can provide important clues to the neural basis of human vocal communication. This is an idea that will be extended to the domains of developmental genetics and molecular neuroscience later in this chapter.

\subsection{Speech Production}

In the majority of mammalian species, and in human infants, the high position of the larynx in the throat allows it to be engaged into the nasal passages, such that the animal can breathe and swallow (or suckle) at the same time (25). During the first 3-4 yr of human life, the larynx undergoes a gradual descent, and its adult location is notably lower than that found in adults of the other primates, including our closest relative, the chimpanzee. This arrangement enables the human tongue to make a greater variety of movements, both horizontally and vertically, within the vocal tract. The concomitant widening of the repertoire of possible vocal-tract shapes results in a dramatically expanded range of distinguishable speech sounds (phonemes) that can be produced (28). There is no doubt that the wider phonetic repertoire conferred by this laryngeal descent is an important feature of human communication, and that its evolution was a key step for emergence of speech and language. What is less clear is why this unusual vocal tract anatomy evolved in the first place (25). Evidence has recently emerged for independent evolution of a descended larynx in some other mammalian species (including red and fallow deer), indicating that this kind of configuration can evolve for reasons other than increased phonetic diversity (29). One suggested explanation is that the vocalizations of an animal with an elongated vocal tract may convey the impression to the listener that the animal is larger than it really is, and this ability to exaggerate size could provide a selective advantage (29).

Regardless of the original reasons for evolution of a descended larynx in humans (among other anatomical differences that are not discussed here), a salient point is that the distinctive vocal-tract anatomy of humans, as compared with other primates, must be under genetic control; hence, at least a subset of the genes that are 
critical for human speech and language will have little or nothing to do with brain function, but will instead be related to anatomical constraints (21). The anatomical data also imply that even a chimpanzee with a fully humanized brain would still be severely limited in its abilities to converse using human speech and language. Similarly, it is extremely unlikely that geneticists will ever be able to engineer a mouse that could learn to talk, however sophisticated our techniques of manipulation may become, simply because the anatomical constraints of the mouse vocal tract do not allow it. Curiously, anatomical constraints do not seem to be a problem for certain birds, such as parrots, which show remarkable abilities to mimic human speech.

Even with the appropriate vocal tract anatomy, the complexity of human speech places elaborate demands on the neural systems that control movement. The articulatory gestures that underlie speech involve finely coordinated sequences of movements by the tongue, lips, jaws, and so on, closely synchronized with vibrations of the larynx; such feats of motor control are rarely (if ever) required by any other system in the body. As noted by Fitch (25), minutely subtle changes in relative timing of different articulators or onset of laryngeal vibrations are enough to yield dramatic phonetic distinctions; with a simple change in timing of just tens of milliseconds the word 'pat' may be turned into 'bat'. Although it has yet to be established whether nonhuman species are capable of comparable levels of fine motor control, based on current data it seems likely that the evolution of human speech depended to some extent on the honing of such abilities (30). This might have involved peripheral mechanisms (for example higher numbers of motor neurons innervating the muscle fibers of the articulators; ref. 31) or higher-order reorganization in the brain (such as increased connectivity of neural circuitry underlying motor coordination; ref. 30), or a combination of the two. I will revisit the key issue of motor control in Section 4. of this chapter because it has recently become particularly relevant for gene-driven studies of precursors of speech in nonhuman species.

\subsection{Speech Perception}

An effective communication system must possess the property of parity, processes of production must somehow match up with processes for perception if meaning is to be accurately conveyed from one individual to another (27). Indeed, humans are born with capacities that are already well attuned to the properties of speech (26). From $4 \mathrm{~d}$ of age, a human newborn can discriminate phonemes in a categorical manner (32) and can detect differences between languages that have distinctive rhythmic structures, but not if the speech sequences are played backwards (33). However, it has been robustly demonstrated that capacities such as phoneme discrimination and sensitivity to speech rhythm are not actually unique to humans. A wide variety of animal and bird species are able to distinguish different human speech sounds; for example, Japanese quail can be taught to discriminate between consonants /b/, /d/, and /g/ even if they are placed before novel vowel sounds that they have not encountered (34). Moreover, adult tamarin monkeys, similar to newborn humans, can discriminate the distinctive rhythmic properties of 
Dutch and Japanese; an ability that, as for newborn humans, is lost when the speech stream is reversed (35). Of course, this does not mean that monkeys can understand Dutch or Japanese. It is important to appreciate the limitations of this kind of comparative study, which may be open to a number of alternative interpretations (36). For example, the adult tamarins that have been studied received a lifetime of exposure to human speech patterns, whereas the only exposure for the human neonates would have been in the womb (albeit at a period of brain development with a dramatic level of plasticity), and the underlying mechanisms for rhythm discrimination might be radically different in the two species (36). Nevertheless, this work does indicate that we should not simply assume that perceptual capabilities attuned to properties of speech are necessarily unique to humans, even if they do appear very early in development.

\subsection{Syntactical Processing}

Currently, the best candidate for a qualitatively distinct process that makes a unique contribution to human vocal processing is the capacity for generating an unlimited array of meaningful utterances using a finite set of lexical units, and a system of rules (syntax) regarding how they can be combined (1-3). At the cognitive level, syntactical processing is able to act independently, as illustrated by Noam Chomsky's much quoted sentence "colourless green ideas sleep furiously," which can be easily judged as grammatically correct even though it lacks any real meaning (1-3). Compare this with another example with the same words in a different order, such as "furiously sleep ideas green colourless," which is obviously ungrammatical. During the first few years of life, a human child is exposed to only a tiny subset of possible sentences, but is nevertheless able to extract correctly the syntactic rules that are inherent to his or her native language (1-5). The use of hierarchical rules (in which one phrase can be embedded within another) is a core feature of syntax that gives it enormous generative power $(2,3,15)$. It has been proposed that monkeys are able to learn simple rule systems, but that, among the primates, the ability to perform abstract hierarchical processing is only found in humans (15). It remains to be seen whether this ability is seen in primitive form anywhere else in the animal kingdom. Finally, it is worth noting that syntactical processing is, in large part, a question of assembling sequences of language elements, and may, thus, be closely related to motor sequencing; interestingly, classic language regions of the brain, such as Broca's area, have been implicated both in syntactical processing and motor sequencing (30).

\section{THE HUMAN FOXP2 GENE: A FOOT IN THE DOOR}

The bulk of the evidence from comparative studies supports a view in which many of the processes underlying human vocal communication exploit neural substrates that were already present (at least in primitive form) in our nonspeaking ancestors. Such a perspective predicts that elucidation of neuromolecular mechanisms in animals will provide an important basis for understanding the potential 
roles of genetic factors in human speech and language. We are now in a position to evaluate this prediction directly. As outlined in the introduction, studies of human language-related disorders are promising to deliver genes that are implicated in the process of speech and language acquisition (16-21). FOXP2, the first of these genes to emerge, provides compelling empirical confirmation of the relevance of ancestral neurogenetic pathways for modern human vocal communication.

\subsection{What is FOXP2?}

The FOXP2 gene encodes a protein belonging to the forkhead family of transcription factors $(20,37)$, so-called because mutations of the founding member in fruit flies cause homeotic transformation of terminal portions of the gut into ectopic head structures (38). The human genome encodes more than 40 different forkhead proteins, each of which includes a highly conserved DNA-binding domain (the forkhead-box or FOX domain) of 84-110 residues (37). All forkhead proteins seem to be activators or repressors of gene transcription, but they can play many diverse roles at cellular and developmental levels, influencing cell cycling, signaling, differentiation, apoptosis, and so on, with some proteins having multiple functions in different tissues or at different times (37). Many forkhead proteins influence developmental pathways during embryogenesis, and mutations in the genes encoding them are known to cause a variety of inherited human and mouse disorders, including glaucoma, immune deficiency, ovarian failure, diabetes, and hearing impairment (39). Alterations in dosage (the number of functional gene copies) of individual forkhead genes can perturb development in a striking manner $(37,39)$. For example, heterozygous missense and nonsense mutations of $\mathrm{FOXCl}$ have been found in subjects with autosomal dominant eye disorders; functional and structural analyses indicate that the mutant $\mathrm{FOXCl}$ alleles lead to loss-of-function of the encoded protein (40), supporting a haploinsufficiency mechanism, i.e., the quantity of functional protein made from a single gene copy is insufficient to allow normal development.

FOXP2 is a member of a specific sub-branch of forkhead proteins (FOXP1-4), which are defined by an unusual variant of the DNA-binding domain spanning only 84 residues, as compared with the more than 100-residue domains usually found in other forkhead proteins (Fig. 1) (20,41-43). Outside the distinctive DNA-binding domain, the FOXP proteins share a glutamine-rich C-terminus, and a highly conserved region containing a zinc finger and a leucine zipper (20,41-43). The latter mediates homodimerization and heterodimerization, which seem to be necessary for DNA-binding and transcription factor function $(42,43)$. The requirement for dimerization is another feature that makes these proteins distinct from other forkheads, most of which are thought to act as monomers (37).

\subsection{Why is FOXP2 Relevant for Human Speech and Language?}

Point mutations in the human form of FOXP2 (Fig. 1), which maps to cytogenetic band 7q31, cause a rare monogenic form of speech and language disorder that is inherited in an autosomal dominant manner $(20,44-46)$. In one well-studied 


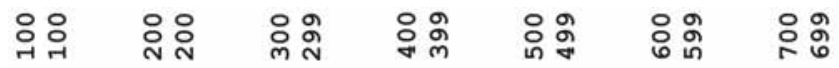

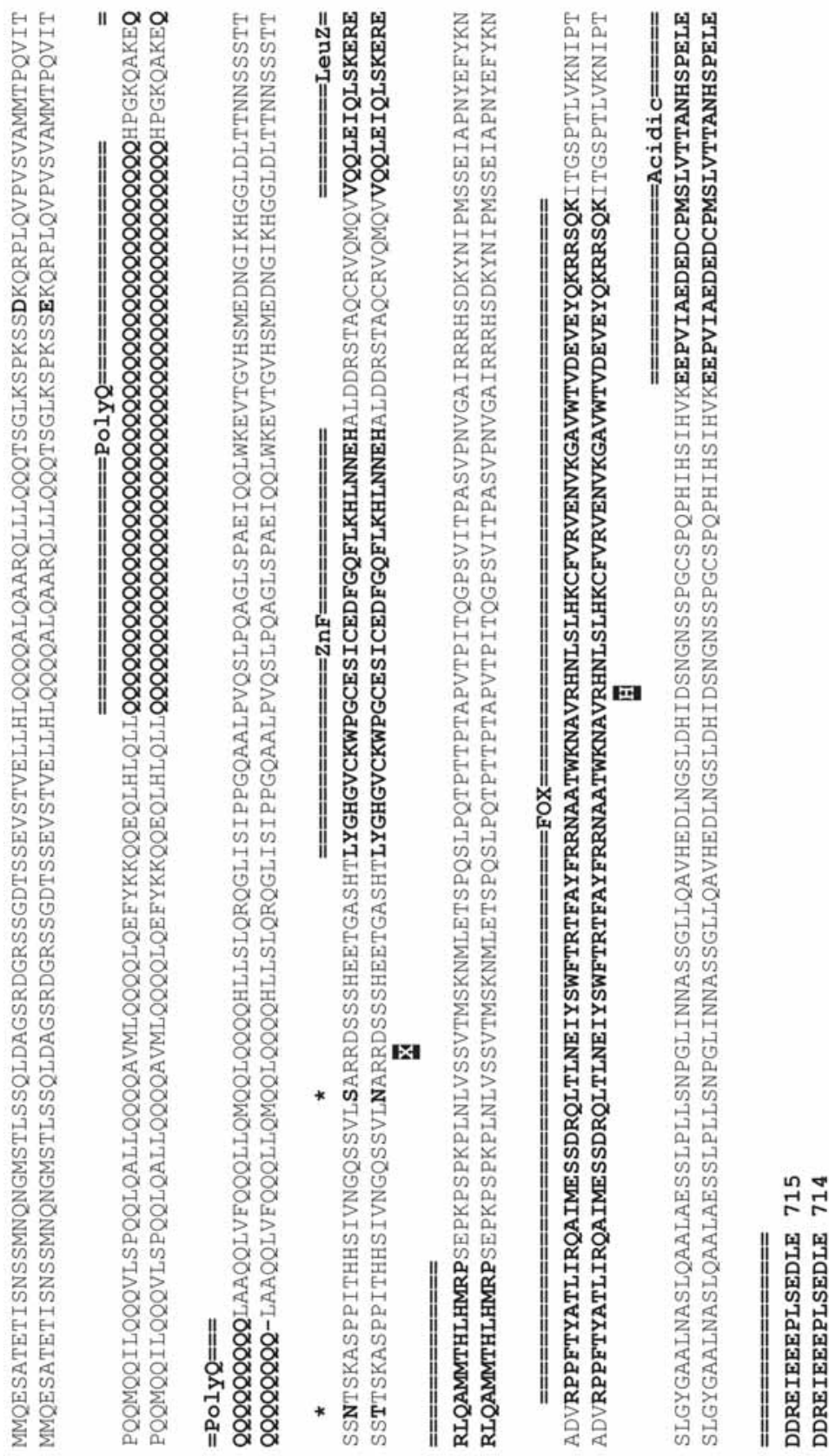

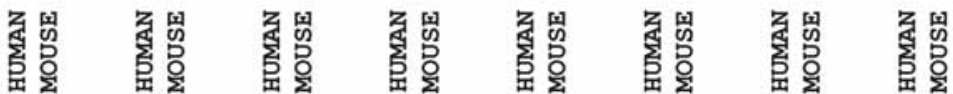


family, known as KE $(44,45)$, a heterozygous missense mutation altering a highly conserved residue in the DNA-binding domain (arginine-to-histidine; R553H) was found to co-segregate with disorder in 15 affected individuals across three generations (20). The mutation was not present in any unaffected members of the family, or in a large panel of normal controls (20). An equivalent R-to-H substitution at the corresponding position in the DNA-binding domain of another human forkhead protein, FOXC1, causes a developmental eye disorder, and in vitro assays indicate that it dramatically disrupts function (40). Thus, there is strong evidence that the $\mathrm{R} 553 \mathrm{H}$ change is of etiological significance for the KE family. In an unrelated small nuclear family with similar impairment in speech and language abilities, a heterozygous nonsense mutation (R328X) was found in two affected siblings, as well as their mother, who had a history of speech problems (46). The mutation, which was not detected in screening of more than 250 control chromosomes, leads to severe truncation of the product encoded by this allele (Fig. 1). The resulting FOXP2 protein is predicted to lack essential functional motifs, including the entire zinc finger/leucine zipper region and forkhead-box domain, and is, thus, unlikely to be able to dimerize or bind to DNA (46). Moreover, the resulting FOXP2 protein has lost critical nuclear localization signals, and, thus, may be inappropriately targeted to the cytoplasm, further hindering its function.

In addition to these mutations, gross chromosomal rearrangements disrupting $F O X P 2$ have been identified in isolated cases of speech and language delay. For example, the FOXP2 locus is directly interrupted by a chromosomal breakpoint in an affected child with a de novo balanced translocation involving chromosomes 7 and $5(20,47)$, and is hemizygous in cases of disorder associated with interstitial deletion encompassing 7q31 $(48,49)$. Thus, the evidence from point mutations and chromosomal abnormalities indicate that loss-of-function of one copy of the FOXP2 gene leads to developmental deficits in speech and language acquisition. Based on these findings, it is likely that FOXP2-associated disorder results from a mechanism of reduced functional gene dosage during early development.

Fig. 1. (opposite page) Alignment of amino acid sequences of human and mouse FOXP2/ Foxp2 proteins (main isoform), as inferred from complementary DNA sequence. Key protein domains include polyglutamine tracts (PolyQ), a zinc-finger motif ( $\mathrm{ZnF})$, a leucine zipper (LeuZ), a forkhead domain (FOX), and a C-terminal acidic region. Disease mutations: two independent point mutations causing speech and language disorder have been identified in the human FOXP2 gene; one leads to an R553H substitution in the forkhead domain, the other truncates the protein at R328, yielding a severely truncated product lacking $\mathrm{ZnF}$, LeuZ, FOX, and acidic domains. The positions of the changes caused by these two mutations are indicated by shaded text beneath the alignment. Comparative genomics: the second polyglutamine tract of the mouse protein is one residue shorter than that in human. Elsewhere, only three amino acids differ between human and mouse (indicated by $*$ above the alignment); none of these occur in known functional domains. Two of these changes (in the region upstream of the $\mathrm{ZnF}$ domain) are specific to the human lineage. See Section 4.3. for further details. 


\subsection{Insights From Humans: Defining the Phenotype}

Human speech and language depend on a complex system of interconnected sensory, motor, and cognitive neural substrates. Impaired acquisition of speech and language skills might potentially result from a range of different abnormalities at the neural level. As such, it is important to probe carefully the phenotypic profile of a disorder to shed light on this issue, and to establish whether impairment is confined to language systems or extends into more general domains. Regarding the disorder caused by disruption of FOXP2, the vast majority of evidence has so far come from the KE family (44,50-57), which may limit the conclusions that can be drawn, but data are now beginning to surface from other cases (49). An early, muchpublicized report proposed that affected subjects in the KE family suffered from a specific deficit in syntactical processing (50), such that this disorder might, for the first time, give a genetic handle to a uniquely human cognitive subprocess (1). However, detailed phenotypic evaluations carried out over the past decade cast doubt on this characterization (51-57). Rather than involving just one aspect of syntax (50), the FOXP2-associated disorder broadly affects a range of languagerelated skills, impacting both the production and comprehension of language $(51,52,55)$. Moreover, in a subset of affected subjects within the KE family, language impairment is accompanied by a less significant reduction in nonverbal abilities (51), although the evidence suggests that these nonverbal deficits are not direct effects of FOXP2 disruption (55). It has been generally established that presence of language impairment during early development puts children at higher risk of wider cognitive deficits and behavioral problems later in life $(58,59)$.

In fact, the most overt feature of disorder transmitted through the KE family, and one that unambiguously distinguishes between affected and unaffected subjects, is a persistent problem in coordinating sequences of mouth movements underlying speech $(44,52,54)$. This kind of articulatory impairment is often referred to as developmental verbal dyspraxia, and it also affects production of nonspeech sounds $(52,54)$. Notably, etiological variants of FOXP2 have not yet been found in children with typical forms of autism, specific language impairment, or dyslexia (60-63), which are all language-related disorders that do not usually involve verbal dyspraxia as a primary feature. In contrast, FOXP2 coding changes, although still a rare cause of impairment, may be present in up to $6 \%$ of children in whom verbal dyspraxia is the most prominent aspect of disorder (46).

It remains open to question whether all of the wider deficits in language ability in affected subjects stem from a primary deficit in articulation (21). An attractive alternative hypothesis is that a single core deficit in learning/production of sequences is directly responsible both for problems with speech and impairment in grammatical processing (53). A third possibility is that FOXP2 disruption simultaneously impacts multiple different neural substrates to yield the wide spectrum of language problems in affected subjects. It is worth noting here that the linguistic dysfunctions observed in these subjects are not confined to oral output (which is obviously strongly influenced by articulatory difficulties), but are also apparent in 
written language (55). More detailed discussion of issues regarding the phenotypic profile of the KE family can be found elsewhere $(17,21)$.

\subsection{Insights From Humans: Brain Imaging Studies}

The brains of affected members of the KE family seem to be radiologically normal (52). However, detailed structural neuroimaging of multiple individuals from this family has revealed several sites in which the density of gray matter is significantly different between affected and unaffected members (52,56). Because the disorder is developmental in nature, it was hypothesized that structural pathology must be present in both hemispheres; otherwise the early plasticity of the brain would allow the subjects to compensate by recruiting circuitry in the unaffected hemisphere $(52,56)$. Damage to just one hemisphere in young children rarely leads to language impairment, because of the ability of the brain to reorganize during early development. Notably, a bilateral reduction in gray matter density has been found in the inferior frontal gyrus (which contains Broca's area, a classic language region of the brain), the caudate nucleus, the precentral gyrus, the temporal pole, and the cerebellum $(52,56)$. Other regions, including the posterior superior temporal gyrus, angular gyrus, and putamen, have been shown to have bilateral increases in density $(52,56)$.

These kinds of neuroanatomical studies can help to point to potential sites mediating dysfunction in subjects with FOXP2 disruption, but they are not direct indicators of abnormalities in neurological mechanisms. In recent years, functional magnetic resonance imaging (fMRI) approaches have been used to ask whether the brains of affected members of the KE family are able to process language in a comparable way to their unaffected relatives (57). In normal right-handed individuals, a task involving generation of verbs in response to aurally presented nouns leads to activation of Broca's area in the left hemisphere, which can be clearly detected using fMRI. For members of the KE family who have the R553H FOXP2 change, there is significant underactivation of Broca's area, and other languagerelated regions, when carrying out this verb generation task. Instead, these subjects show diffuse bilateral activation of regions of the cortex that are not usually associated with speech- or language-based tasks (57). The affected subjects are able to perform the task adequately, but the pattern of neural activation seems to be highly atypical, suggesting that they may be compensating for the underlying genetic deficit to a certain extent via reorganization of neural circuitry during development (a common feature for neurodevelopmental disorders such as dyslexia and specific language impairment) $(18,19)$. For the overt scenario, in which test subjects are asked to give spoken responses during the verb generation task, the underactivation of Broca's area in affected KE family members may be simply related to abnormalities in execution of speech output, because this region of the brain is also implicitly involved in motor sequencing (30). Therefore, perhaps this finding might be explained purely in terms of peripheral speech mechanisms. However, the same underactivation was observed for covert verb generation, in which the subjects were 
instructed to think their responses but not produce any speech output (57). Thus, these fMRI experiments provide the strongest support so far for the hypothesis that FOXP2 disruption leads to abnormalities in the neural circuitry underlying language processing (specifically, semantic retrieval and articulatory planning), rather than impacting only on domain-general mechanisms involved in controlling fine muscle movements. Subcortical regions of the brain, particularly areas of the striatum, have also been implicated by functional imaging studies. Thus, both structural and functional approaches indicate that there is distributed pathology associated with FOXP2 disruption, involving cortical and subcortical structures, as opposed to damage of one specific focal region of the central nervous system (CNS) $(49,52,56,57)$.

\section{FOXP2 IN ORGANISMS THAT LACK LANGUAGE}

Studies of human subjects with FOXP2 disruption have been of great importance for increasing understanding of the nature of the resulting disorder, but they hit limitations on two separate fronts. First, mutation of FOXP2 is extremely rare in the human population, therefore, conclusions must be drawn from extensive testing of very small numbers of individuals; as stated above in Section 3.3., almost all phenotypic and neuroimaging investigations have thus far focused on only one allelic variant, which is found in just a single pedigree (R553H in the KE family) (50-57). Second, there are (at least currently) severe practical and ethical restrictions to the scope of human-based research, precluding the possibility of proper in vivo investigations of human CNS function at the neuromolecular level. Unfortunately, investigations of gene function in human neuronal-like cell lines, although certainly of value, are not able to model the complexities and subtleties of brain architecture and function. A tried and tested solution to this problem is to gain insights into function of the human gene by studying orthologs found in other species, with techniques that enable investigation and correlation of data at multiple levels (anatomical, developmental, molecular, and so on). However, if human FOXP2 is intimately involved in speech and language acquisition, aspects of our make-up that are supposedly unique to our species, then what could the orthologs of this gene be doing in nonlinguistic species? Although the full answer to this question is not yet known, a number of provocative clues have already emerged.

\subsection{Foxp2 in Rodents}

A naïve perspective of "speech genes" might predict that FOXP2 would not be present in the genomes of nonlinguistic species, or that it may differ greatly in sequence, expression, or function. Studies of mammalian orthologs of FOXP2 have contradicted this simplistic view in a spectacular manner. In terms of coding sequence, the FOXP2 gene represents one of the most highly conserved loci in the evolutionary history of humans and rodents (Fig. 1) $(64,65)$. Of the 715 amino acid residues in the main isoform of FOXP2, the mouse version differs at only four positions (three amino acid substitutions, and a single residue reduction in length of 
one of the polyglutamine tracts). In other words, the mouse protein is identical in sequence to the human ortholog for approx $99.5 \%$ of its length. Moreover, the amino acid substitutions lie outside the currently known functional domains of the protein, thus it is not clear what impact they might have on behavior of this transcription factor.

Could the presence of a highly conserved version of Foxp2 in nonspeaking rodents be explained in terms of a role or roles outside the CNS, in the development or functioning of other tissues? In part, the answer to this question is yes. During embryogenesis, murine Foxp2 is expressed in defined regions of multiple tissues, including the distal alveolar lung epithelium, the outer mesodermal intestinal layer, and the outflow tract and atrium of the cardiovascular system (41). Studies have shown that the Foxp2 protein is able to repress lung-specific target genes in vitro (41-43). Similarly, human FOXP2 is expressed in multiple tissues during fetal development and in adulthood (20). The recruitment of the same transcription factor to multiple pathways in different tissues and at distinctive times in the life of an organism is a characteristic feature of this class of proteins (37), thus, FOXP2 is not at all unusual in this respect. Therefore, FOXP2 is indeed likely to have evolutionarily conserved roles that have no relation whatsoever to brain function or speech. However, when we look to expression patterns that are found in the CNS, the story becomes even more complex, and more intriguing.

In mice, rats, and humans, Foxp 2/FOXP2 messenger RNA (mRNA) is expressed in neurons (but is not detected in glial cells) in a variety of different brain structures during embryogenesis (66-68), supporting a possible role in aspects of early neurodevelopment. In the same way that disruption of FOXP2 in humans does not lead to damage to just one specific region of the brain, expression of the gene is not confined to any one CNS structure. In fact, significant levels of mRNA and protein have been detected in multiple regions of the forebrain, midbrain, and hindbrain (66-68). However, the peculiar spatiotemporal expression patterns found in each region indicate that this gene is subject to very precise regulation and give hints regarding potential mechanisms that FOXP2 may be controlling during neurodevelopment. Three cases of sublocalization merit discussion:

1. In the developing and mature cortex, Foxp 2 expression is restricted to the deepest layers (mainly layer 6), consisting of early-born pyramidal neurons that project back to subcortical regions, such as the dorsal thalamus, also a site of Foxp2 expression (66-68). Other transcription factors, such as Tbrl and Otxl, show similar deep layerspecific (or enriched) expression patterns in the mammalian cortex; mutation of genes encoding these transcription factors results in disruption to corticothalamic connectivity $(69,70)$.

2. A well-documented subcortical site of high FOXP2/Foxp2 expression is the striatum (consisting of the caudate nucleus and the putamen), with levels that are highest during embryogenesis but persist throughout development (66-68). In rats, Foxp2 shows differential postnatal expression in the two different compartments that make up the striatum, which are known as the striosomes and the matrix (68). These compartments are distinguished by differing profiles of expression for receptors, neurotransmitters, signaling molecules and so on, and by distinct neurodevelopmental origins and pat- 
terns of connectivity with other brain regions (71). Rat Foxp2 expression seems to be restricted to the striosomal compartment throughout the life of the animal (68), although it has not been determined whether this observation applies to other mammalian species. Studies have shown that perturbations in the balance between striosomes and matrix can impact motor behavior (72), which may be relevant to the motor aspects of the phenotype associated with human FOXP2 disruption.

3. The cerebellum is a complex multilayered structure in the hindbrain with many different cell types, including granule, Golgi, basket, stellate, and Purkinje cells. In this brain region, FOXP2/Foxp 2 is detected specifically in Purkinje cells and deep cerebellar nuclei. In addition, strong persistent expression is found in the inferior olive (67), a precerebellar nucleus providing direct input to these neurons. During late embryogenesis, climbing fibers from the inferior olives innervate Purkinje cells (73) and organize their topography in an ordered fashion, perhaps in response to polarity cues (74).

Overall, the expression studies that have been conducted thus far in mammalian species support two key conclusions. First, the spatiotemporal patterns observed in humans and rodents are strikingly similar (Fig. 2). Although the human studies are inevitably more limited in scope because of restricted availability of CNS tissue at later stages of embryonic development, such that some potential expression differences may be missed, the regulation of FOXP2/Foxp 2 at comparable stages is remarkably concordant in different species (67). Second, the expression data are most compatible with this gene having a role (or roles) in establishing and/or maintaining neural circuitry involved in motor control and sensorimotor integration. In particular, corticostriatal and olivocerebellar circuits (key sites of FOXP2/Foxp2 expression identified by multiple studies) are fundamental for motor control; the basal ganglia modulate activity of premotor and prefrontal cortex via complex connections projecting through the globus pallidus, substantia nigra, and thalamus (75), and the Purkinje cells of the cerebellum play a key role in regulating motor coordination, receiving strong synaptic excitation from inferior olivary climbing fibers (76). Of note, there is growing appreciation that circuits involving striatal and cerebellar structures are not limited to motor function, but are also essential for aspects of cognition, underpinning aspects of many complex learned behaviors $(30,75)$.

\subsection{Foxp2 in Birds}

Although humans are the only organisms known to speak, we are not alone in our capacity to acquire new vocalizations via imitation of our peers. This ability (known as vocal learning) is nevertheless an extremely rare trait among animals and birds, found only in a handful of species, in contrast to the more pervasive use of innately specified calls (such as alarm calls made on encountering a predator) (77). The only known vocal learning species are found in three groups of birdsparrots, hummingbirds, and songbirds - and three groups of mammals-humans, cetaceans (whales and dolphins), and bats. In both birds and mammals, different vocal-learning species are phylogenetically separated from each other by nonlearners, suggesting that there may have been independent evolution of this trait on at least six different occasions (77). The human communication system can be viewed as a highly specialized form of vocal learning; acknowledging that the 


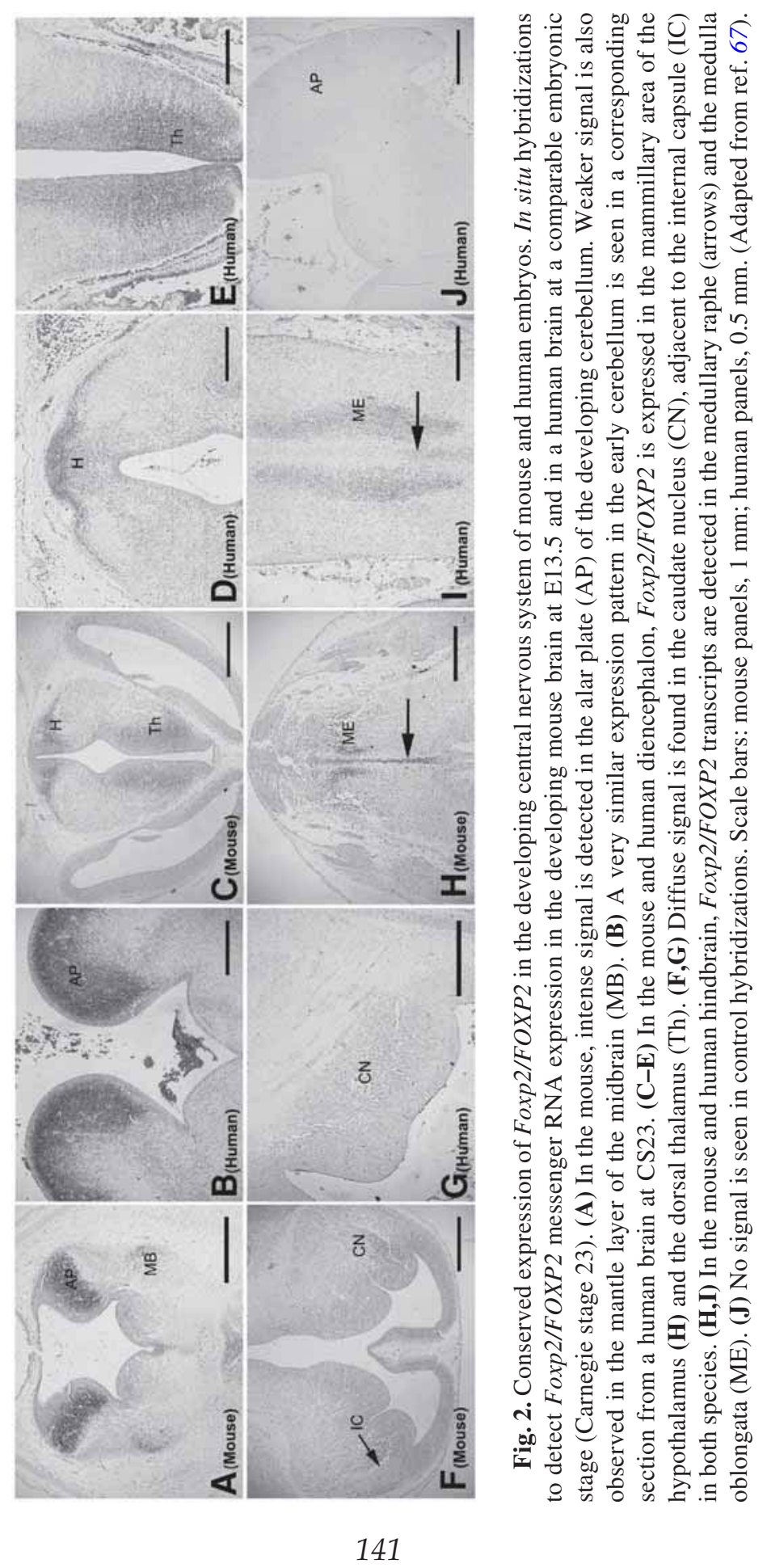


communication skills of parrots, hummingbirds, and songbirds are not as sophisticated as human language (for example it is not known whether avian vocal learners have the capacity to use hierarchical systems of syntax).

Neuroanatomical and functional genetic investigations of different bird species have indicated the presence of seven discrete forebrain structures involved in vocal control (learning and production), which seem to be common to all vocal-learners. These so-called song nuclei are absent from the brains of nonlearning birds, or perhaps present in such a rudimentary form that they have escaped detection (77). Elegant neurobiological studies demonstrate that gene expression patterns in the song system change during song learning and production $(78,79)$. If the song nuclei are indeed unique to vocal learners, then one explanation would be the independent evolution of a set of seven similar forebrain structures on three separate occasions in the ancestry of modern birds, thus indicating severe epigenetic constraints on evolution of vocal control structures. Conversely, the song system may have been present in the common ancestor of all avian vocal learners, with loss of the entire set of forebrain control structures on four separate occasions during bird evolution. The maintenance of a vocal learning system in the brain places considerable demands on an organism's resources, and thus may only represent a selective advantage in certain types of environment (77). Regardless of these evolutionary conundrums, which are yet to be resolved, studies of birds offer a unique window on neurogenetic mechanisms involved in vocal learning, and the resulting insights may be generally relevant for our understanding of this trait in the form that is found in our own species (79). Notably, the anterior nuclei of the song system participate in neural pathways comparable to mammalian corticostriatal circuitry, active in the learning and maintenance of motor sequences dependent on sensorimotor integration (77-79). Thus, given the emerging evidence that mammalian FOXP2 may be involved in motor-related circuitry, it becomes of immediate interest to evaluate the potential role of avian FoxP2 in the development and/or behavior of the song system.

Two recent studies have made the first attempts to address this issue $(80,81)$. FoxP2 in songbirds is very similar to the human and rodent orthologs; the zebra finch protein sequence is approx $98 \%$ identical to the mammalian proteins. Moreover, for this gene there is a remarkable concordance in the spatiotemporal CNS expression patterns found in birds and mammals. High levels of FoxP2 mRNA and its protein product have been robustly detected in the striatum, dorsal thalamus, Purkinje cells, and inferior olives, with weaker expression in a number of related brain nuclei $(80,81)$. This pattern has been found to be consistent in eleven different species of bird, regardless of sex or song-learning ability (81). As in mammals, expression starts early in embryogenesis, and persists through development and into adulthood. The levels of expression in these regions are comparable across multiple avian species, but when it comes to vocal control structures (only present in vocal learners), FoxP2 expression seems to show species-specific differences, which might be related to variability in vocal plasticity $(81)$. For example, in zebra 
finches, the striatal nucleus known as Area X shows higher FoxP2 levels than that found in surrounding tissue, but only at the point in development when the birds are learning to imitate song. In adult canaries, Area X expression seems to vary with the season; FoxP2 levels are highest in the months when song shows most plasticity.

Considered together, the data from humans, rodents, and birds clearly support an ancient role for this gene in establishing and maintaining sensorimotor circuitry of the CNS in all vertebrates, regardless of vocal-learning abilities. Thus, it has been suggested that the original CNS function of FoxP2 was not specific for vocal learning, but that it created a so-called permissive environment in the brain, on which vocal learning might evolve, conditional on other factors (82). Modification and elaboration of sensorimotor-related circuitry is likely to have been a key feature in the independent evolution of complex learned behaviors in different species, including the development of song learning in subsets of avian species and the acquisition of a capacity for speech and language in humans. It should be stressed that this argument does not assume a direct equivalence for birdsong and human language, but is, nevertheless, based on striking parallels in these differing vocal communication systems.

\subsection{Foxp2 in Primates}

In contrast to the wealth of expression data already accumulated for the CNS of birds and rodents (and even human embryos) little is known about the spatiotemporal patterning of Foxp 2 during brain development of nonhuman primates. Investigations of the gene in nonspeaking primates have approached the question of species difference from another direction, that of comparative DNA sequencing $(64,65)$. These studies revealed that, despite the notable conservation in protein sequence of FOXP2 and its orthologs in multiple vertebrate species, something peculiar seems to have happened during primate evolution. Specifically, out of the three amino acid substitutions that differentiate between the rodent and human orthologous proteins, two seemed to have occurred on the lineage that led to modern Homo sapiens, after it split from the lineage leading to chimpanzees (Fig. 1) (64,65). In other words, regarding the sequence of the FOXP2 protein, a chimpanzee is more similar to a mouse than to a human, even though the evolutionary distance that separates chimpanzees and mice is much larger than that between chimpanzees and humans. Examination of noncoding intronic sequences in the vicinity of the exon carrying the two human-specific amino acid substitutions suggested that these changes had been subject to positive selection on the human lineage, probably within the last $200,000 \mathrm{yr}(64,65)$. Although these data might point to some functional impact for one, or both, of the amino acid changes in question, with respect to recently evolved human traits (perhaps relating to speech), there is, as yet, no corroborating data from protein studies. In fact, the changes occurred in a region of FOXP2 that is little understood, outside the known domains, and, although one of them creates a potential phosphorylation site that could affect posttranslational regulation, the same change occurred independently during the evolution of carnivores (65). 
Because data from evolutionary anthropology suggest that human speech evolved in the past 200,000 $\mathrm{yr}$ (83), it might be tempting to speculate that positive selection of amino acid substitutions of FOXP2 was the sole driving force for the appearance of a functioning human vocal communication system. In fact, such a scenario is highly unlikely, especially given what we now know about the conserved ancestral functions of the gene in the vertebrate CNS. Instead, it is more appropriate to view the changes in this gene as one element in a complex network of events that led to our modern speech and language capacity. The picture that is emerging is one in which Foxp2 was already playing a role in the common ancestor of humans and rodents, probably in patterning of sensorimotor neural circuitry underpinning behaviors such as sensory-mediated learning of motor sequences. At a time when vocal learning was becoming important in human society, modifications to the gene that improved such behaviors could have rapidly spread through the population by positive selection and become fixed. This hypothesis is similar to the idea that FoxP2 helped to provide a permissive neural environment for vocal learning in avian species (82), although there are no reports demonstrating evidence for positive selection of the gene in avian evolution.

\section{TALKING TO THE ANIMALS}

The FOXP2 gene has provided us with the first glimpse into neurogenetic mechanisms that contribute to our species' capacity for speech and language. It is impossible to say at this stage whether other genes related to human vocal communication will be akin to this example, but the FOXP2 story suggests an optimistic outlook for animal studies in this area. Studies of expression patterns in nonspeaking species have already yielded critical new insights into the potential role of this gene in the brain $(66-68,80,81)$, and indicate that the available technologies for working with model organisms will be powerful for future research into the gene's function. A particularly valuable approach will be to carry out targeted knockouts of the Foxp 2 gene in mice. These studies are already underway by a number of different research groups. It would be absurd to suggest that this will create a murine model of human speech and language disorder, although there is certainly no question that the resulting mice would be unable to speak. Instead, these mice will provide molecular, developmental, anatomical, and behavioral insights into gene function that might never be revealed by studies of humans, and they will facilitate approaches for establishing in vivo targets and interaction partners of the gene in question.

What exactly might scientists be able to discover from mice with a Foxp2 disruption? From a molecular neuroscience perspective, it will be possible to use methods such as birth dating of neurons, tracing of axonal tracts, and labeling with region-specific markers (e.g., refs. 69,70) to discover precisely how Foxp2 influences the development of corticostriatal and olivocerebellar circuitry in the mammalian CNS. These techniques might answer questions such as: is the gene involved in proliferation, migration, differentiation, axon growth, or cell death? The expression data suggests a postmigratory function in a number of CNS regions, perhaps in 
connectivity (66-68). Does it have distinct roles in different regions and at different times? Does reduced Foxp 2 dosage in mice disrupt development in all of the known regions of expression, or just a subset? What happens to striosome/matrix compartmentalization $(71,72)$ or ordering of olivocerebellar connections $(73,74)$ when Foxp 2 dosage is altered? None of these questions can be reliably addressed by studying humans. At the anatomical level, it will be interesting to determine whether structural anomalies observed in humans with FOXP2 disruption (such as the reduction of gray matter in the caudate nucleus; ref. 56) might be similarly present in mutant mice, and, if they are, for the first time, it will be possible to establish the molecular and cellular bases of this finding. For behavioral studies, it will be feasible to test whether abnormalities in Foxp2 have consequences for complex learned behaviors in nonspeaking species. We cannot hope to ever study linguistics and grammar in mice with Foxp2 disruption, but this is something that can be very effectively studied in humans.

Of course, knockout studies have their own well-documented limitations; for example, phenotypic consequences of gene disruption may be highly dependent on modifier genes in the background, or on environmental factors. One common problem can be that genes such as Foxp2, which are normally expressed in multiple tissues, may lead to embryonic lethality when disrupted (84). Conditional techniques in which the gene is knocked out in only a subset of tissues, or at a specific point in development (84), will ameliorate this in many cases, although not always. Conditional targeting might also allow a dissection of region-specific roles of Foxp2 in the brain, by disrupting the gene in some CNS structures, while allowing it to be normally expressed in others. The bottom line is that we cannot expect to obtain all the answers from just one approach. An understanding of the way that FOXP2 impacts vocal communication must rely on integration of data from multiple distinct types of endeavor, but it is crucial that animal work is one of these.

In conclusion, studies of the first known example of a gene that influences speech and language development indicate that key aspects of human vocal communication involve modifications of neurogenetic mechanisms that were already present in our nonspeaking ancestors. It remains possible that other genes exist with functions that are confined to our species, but the discovery of FOXP2 illustrates that this need not be the case for all language-related genes. Consequently, investigations of such genes in animals promise to be highly fruitful for our future understanding of the neural basis of speech and language. FOXP2 itself is likely to influence development of cortical and subcortical circuits involved in sensorimotor functions, not just in humans, but also in all vertebrates. A comprehensive account of the neuromolecular pathways regulated by the ancestral form of this gene is a prerequisite for gaining insight into the role of modern human FOXP2 in speech and language development.

\section{ACKNOWLEDGMENTS}

Dr Fisher is a Royal Society Research Fellow. His research is also funded by project grants from the Wellcome Trust and the Medical Research Council. 


\section{REFERENCES}

1. Pinker S. The Language Instinct. London, UK: Allen Lane, 1994.

2. Chomsky N. Aspects of the Theory of Syntax. Cambridge, MA: MIT Press, 1965.

3. Chomsky N. Rules and Representations. Columbia University Press, New York, NY, 1980.

4. Saffran JR, Aslin RN, Newport EL. Statistical learning by 8-month-old infants. Science 1996;274:1926-1928.

5. Marcus GF, Vijayan S, Bandi Rao S, Vishton PM. Rule learning by seven-month-old infants. Science 1999;283:77-80.

6. Peña M, Bonatti LL, Nespor M, Mehler J. Signal-driven computations in speech processing. Science 2002;298:604-607.

7. Damasio AR. Aphasia. N Engl J Med 1992;326:531-539.

8. Dronkers NF. A new brain region for coordinating speech articulation. Nature 1996;384:159-161.

9. Poeppel D, Hickok G. Towards a new functional anatomy of language. Cognition 2004;92:1-12.

10. Wise RJ, Greene J, Buchel C, Scott SK. Brain regions involved in articulation. Lancet 1999;353:1057-1061.

11. Musso M, Moro A, Glauche V, et al. Broca's area and the language instinct. Nat Neurosci 2003;6:774-781.

12. Peña M, Maki A, Kovačić D, et al. Sounds and silence: an optical topography study of language recognition at birth. Proc Natl Acad Sci USA 2003;100:11,702-11,705.

13. Terrace HS, Petitto LA, Sanders RJ, Bever TG. Can an ape create a sentence? Science 1979;206:891-902.

14. Brockelman WY, Schilling D. Inheritance of stereotyped gibbon calls. Nature 1984;312:634-636.

15. Fitch WT, Hauser MD. Computational constraints on syntactic processing in a nonhuman primate. Science 2004;303:377-380.

16. Bishop DVM. Genetic and environmental risks for specific language impairment in children. Phil Trans Biol Sci 2001;356:369-380.

17. Fisher SE, Lai CSL, Monaco AP. Deciphering the genetic basis of speech and language disorders. Ann Rev Neurosci 2003;26:57-80.

18. Fisher SE, DeFries JC. Developmental dyslexia: genetic dissection of a complex cognitive trait. Nat Rev Neurosci 2002;3:767-780.

19. Folstein SE, Rosen-Sheidley B. Genetics of autism: complex aetiology for a heterogeneous disorder. Nat Rev Genet 2001;2:943-955.

20. Lai CSL, Fisher SE, Hurst JA, Vargha-Khadem F, Monaco AP. A novel forkhead-domain gene is mutated in a severe speech and language disorder. Nature 2001;413:519-523

21. Marcus GF, Fisher SE. FOXP2 in focus: what can genes tell us about speech and language? Trends Cog Sci 2003;7:257-262.

22. Zaki PA, Quinn JC, Price DJ. Mouse models of telencephalic development. Curr Opin Genet Dev 2003;13:423-437.

23. Kirik D, Annett LE, Burger C, Muzyczka N, Mandel RJ, Bjorklund A. Nigrostriatal alphasynucleinopathy induced by viral vector-mediated overexpression of human alpha-synuclein: a new primate model of Parkinson's disease. Proc Natl Acad Sci USA 2003;100:2884-2889.

24. Hauser MD, Chomsky N, Fitch WT. The faculty of language: what is it, who has it, and how did it evolve? Science 2002;298:1569-1579.

25. Fitch WT. The evolution of speech: a comparative review. Trends Cogn Sci 2000;4:258-267.

26. Diehl RL, Lotto AJ, Holt LL. Speech perception. Annu Rev Psychol 2004;55:149-179.

27. Liberman AM, Whalen DH. On the relation of speech to language. Trends Cogn Sci 2000;4:187-196.

28. Lieberman PH, Klatt DH, Wilson WH. Vocal tract limitations on the vowel repertoires of rhesus monkey and other nonhuman primates. Science 1969;164:1185-1187.

29. Fitch WT, Reby D. The descended larynx is not uniquely human. Proc R Soc Lond B Biol Sci 2001;268:1669-1675.

30. Lieberman P. On the nature and evolution of the neural bases of human language. Am J Phys Anthropol 2002(Suppl);35:36-62. 
31. Kay RF, Cartmill M, Balow M. The hypoglossal canal and the origin of human vocal behavior. Proc Natl Acad Sci USA 1998;95:5417-5419.

32. Eimas PD, Siqueland ER, Jusczyk P, Vigorito J. Speech perception in infants. Science 1971;171:303-306.

33. Mehler J, Jusczyk P, Lambertz G, Halsted N, Bertoncini J, Amiel-Tison C. A precursor of language acquisition iNYoung infants. Cognition 1988;29:143-178.

34. Kluender KR, Diehl RL, Killeen PR. Japanese quail can learn phonetic categories. Science 1987;237:1195-1197.

35. Ramus F, Hauser MD, Miller C, Morris D, Mehler J. Language discrimination by human newborns and by cotton-top tamarin monkeys. Science 2000;288:349-351.

36. Werker JF, Vouloumanos A. Language. Who's got rhythm? Science 2000;288, 280-281.

37. Carlsson P, Mahlapuu M. Forkhead transcription factors: key players in development and metabolism. Dev Biol 2002;250:1-23.

38. Weigel D, Jurgens G, Kuttner F, Seifert E, Jackle H. The homeotic gene fork head encodes a nuclear protein and is expressed in the terminal regions of the Drosophila embryo. Cell 1989;57:645-658.

39. Lehmann OJ, Sowden JC, Carlsson P, Jordan T, Bhattacharya SS. Fox's in development and disease. Trends Genet 2003;19:339-344.

40. Saleem RA, Banerjee-Basu S, Berry FB, Baxevanis AD, Walter MA. Structural and functional analyses of disease-causing missense mutations in the forkhead domain of FOXC1. Hum Mol Genet 2003;12:2993-3005.

41. Shu W, Yang H, Zhang L, Lu MM, Morrisey EE. Characterization of a new subfamily of wingedhelix/forkhead (Fox) genes that are expressed in the lung and act as transcriptional repressors. J Biol Chem 2001;276:27,488-27,497.

42. Wang B, Lin D, Li C, Tucker P. Multiple domains define the expression and regulatory properties of Foxp1 forkhead transcriptional repressors. J Biol Chem 2003;278:24,259-24,268.

43. Li S, Weidenfeld J, Morrisey EE. Transcriptional and DNA binding activity of the Foxp1/2/4 family is modulated by heterotypic and homotypic protein interactions. Mol Cell Biol 2004;24:809-822.

44. Hurst JA, Baraitser M, Auger E, Graham F, Norell S. An extended family with a dominantly inherited speech disorder. Dev Med Child Neurol 1990;32:347-355.

45. Fisher SE, Vargha-Khadem F, Watkins KE, Monaco AP, Pembrey ME. Localisation of a gene implicated in a severe speech and language disorder. Nature Genet 1998;18:168-170.

46. MacDermot KD, Bonora E, Sykes N, et al. Identification of FOXP2 truncation as a novel cause of developmental speech deficits. Am J Hum Genet 2005;76:1074-1080.

47. Lai CSL, Fisher SE, Hurst JA, et al. The SPCH1 region on human 7q31: genomic characterization of the critical interval and localization of translocations associated with speech and language disorder. Am J Hum Genet 2000;67:357-368.

48. Sarda P, Turleau C, Cabanis MO, Jalaguier J, de Grouchy J, Bonnet H. Interstitial deletion in the long arm of chromosome 7. Ann de Genet 1988;31:258-261.

49. Liègeois F, Belton E, Lai CSL, et al. Comparable behavioural and brain abnormalities in cases with deletion or mutation of the FOXP2 gene, submitted.

50. Gopnik M. Feature-blind grammar and dysphasia. Nature 1990;344:715 [correspondence].

51. Vargha-Khadem F, Watkins K, Alcock K, Fletcher P, Passingham R. Praxic and nonverbal cognitive deficits in a large family with a genetically transmitted speech and language disorder. Proc Natl Acad Sci USA 1995;92:930-933.

52. Vargha-Khadem F, Watkins KE, Price CJ, et al. Neural basis of an inherited speech and language disorder. Proc Natl Acad Sci USA 1998;95:12,695-12,700.

53. Ullman MT, Gopnik M. Inflectional morphology in a family with inherited specific language impairment. Appl Psycholing 1999;20:51-117.

54. Alcock KJ, Passingham RE, Watkins KE, Vargha-Khadem F. Oral dyspraxia in inherited speech and language impairment and acquired dysphasia. Brain Lang 2000;75:17-33. 
55. Watkins KE, Dronkers NF, Vargha-Khadem F. Behavioural analysis of an inherited speech and language disorder: comparison with acquired aphasia. Brain 2002;125:452-464.

56. Belton E, Salmond CH, Watkins KE, Vargha-Khadem F, Gadian DG. Bilateral brain abnormalities associated with dominantly inherited verbal and orofacial dyspraxia. Hum Brain Map 2003;18:194-200.

57. Liègeois F, Baldeweg T, Connelly A, Gadian DG, Mishkin M, Vargha-Khadem F. Language fMRI abnormalities associated with FOXP2 gene mutation. Nature Neurosci 2003;6:1230-1237.

58. Rutter M, Mawhood L. The Long-Term Psychosocial Sequelae of Specific Developmental Disorders of Speech and Language. In: Rutter M, Casaer P, eds. Biological Risk Factors for Psychosocial Disorders. Cambridge, UK: Cambridge University Press, 1991, pp. 233-259.

59. Tallal P, Townsend J, Curtiss S, Wulfeck B. Phenotypic profiles of language-impaired children based on genetic/family history. Brain Lang 1991;41:81-95.

60. Newbury DF, Bonora E, Lamb JA, et al., and the International Molecular Genetic Study of Autism Consortium. FOXP2 is not a major susceptibility gene for autism or Specific Language Impairment (SLI). Am J Hum Genet 2002;70:1318-1327.

61. Wassink TH, Piven J, Vieland VJ, et al. Evaluation of FOXP2 as an autism susceptibility gene. Am J Med Genet 2002;114:566-569.

62. Gauthier J, Joober R, Mottron L, et al. Mutation screening of FOXP2 in individuals diagnosed with autistic disorder. Am J Med Genet 2003;118A:172-175.

63. Kaminen N, Hannula-Jouppi K, Kestila M, et al. A genome scan for developmental dyslexia confirms linkage to chromosome 2 p11 and suggests a new locus on 7q32. J Med Genet 2003;40:340-345.

64. Enard W, Przeworski M, Fisher SE, et al. Molecular evolution of FOXP2, a gene involved in speech and language. Nature 2002;418:869-872.

65. Zhang J, Webb DM, Podlaha O. Accelerated protein evolution and origins of human-specific features. Foxp2 as an example. Genetics 2002;162:1825-1835.

66. Ferland RJ, Cherry TJ, Preware PO, Morrisey EE, Walsh CA. Characterization of Foxp2 and Foxp1 mRNA and protein in the developing and mature brain. J Comp Neurol 2003;460: 266-279.

67. Lai CSL, Gerrelli D, Monaco AP, Fisher SE, Copp AJ. FOXP2 expression during brain development coincides with adult sites of pathology in a severe speech and language disorder. Brain $2003 ; 126: 2455-2462$.

68. Takahashi K, Liu FC, Hirokawa K, Takahashi H. Expression of Foxp2, a gene involved in speech and language, in the developing and adult striatum. J Neurosci Res 2003;73:61-72.

69. Hevner RF, Shi L, Justice N, et al. Tbr1 regulates differentiation of the preplate and layer 6. Neuron 2001;29:353-366.

70. Weimann JM, Zhang YA, Levin ME, Devine WP, Brulet P, McConnell SK Cortical neurons require Otx 1 for the refinement of exuberant axonal projections to subcortical targets. Neuron 1999;24:819-831.

71. Jain M, Armstrong RJ, Barker RA, Rosser AE. Cellular and molecular aspects of striatal development. Brain Res Bull 2001;55:533-540.

72. Canales JJ, Graybiel AM. A measure of striatal function predicts motor stereotypy. Nature Neurosci 2000;3:377-383.

73. Wang VY, Zoghbi HY. Genetic regulation of cerebellar development. Nature Rev Neurosci 2001;2:484-491.

74. Chedotal A, Bloch-Gallego E, Sotelo C. The embryonic cerebellum contains topographic cues that guide developing inferior olivary axons. Development 1997;124:861-870.

75. Middleton FA, Strick PL. Basal ganglia and cerebellar loops: motor and cognitive circuits. Brain Res Brain Res Rev 2000;31:236-250.

76. Welsh JP, Lang EJ, Suglhara I, Llinas R. Dynamic organization of motor control within the olivocerebellar system. Nature 1995;374:453-457. 
77. Jarvis ED, Ribeiro S, da Silva ML, Ventura D, Vielliard J, Mello CV. Behaviourally driven gene expression reveals song nuclei in hummingbird brain. Nature 2000;406:628-632.

78. Jarvis ED, Scharff C, Grossman MR, Ramos JA, Nottebohm F. For whom the bird sings: contextdependent gene expression. Neuron 1998;21:775-788.

79. Brainard MS, Doupe AJ. What songbirds teach us about learning. Nature 2002;417:351-358.

80. Teramitsu I, Kudo LC, London SE, Geschwind DH, White SA. Parallel FoxP1 and FoxP2 expression in songbird and human brain predicts functional interaction. J Neurosci 2004;24:3152-3163.

81. Haesler S, Wada K, Nshdejan A, et al. FoxP2 expression in avian vocal learners and non-learners. J Neurosci 2004;24:3164-3175.

82. Scharff C, White SA. Genetic components of vocal learning. Ann NY Acad Sci 2004;1016: 325-347.

83. Boyd R, Silk JB. How humans evolved. New York: W.W. Norton, 2000.

84. Kuhn R, Schwenk F, Aguet M, Rajewsky K. Inducible gene targeting in mice. Science 1995;269:1427-1429. 